\title{
Atypical Bony Extension in Recurrent Dermatofibrosarcoma Protuberance of Foot
}

\author{
Prerna Patwa ${ }^{1}$, Pratap Singh Hanuman Parihar ${ }^{2}$, Rohan Kumar Singh ${ }^{3}$, Rajasbala Pradeep Dhande ${ }^{4}$ \\ 1Department of Radio Diagnosis, DMIMS, Sawangi (Meghe), Wardha, Maharashtra, India. \\ 2Department of Radio Diagnosis, DMIMS, Sawangi (Meghe), Wardha, Maharashtra, India. \\ ${ }^{3}$ Department of Radio Diagnosis, DMIMS, Sawangi (Meghe), Wardha, Maharashtra, India. \\ ${ }^{4}$ Department of Radio Diagnosis, DMIMS, Sawangi (Meghe), Wardha, Maharashtra, India.
}

\section{INTRODUCTION}

Dermatofibrosarcoma protuberans (DFSP) is a relatively uncommon tumour of the spindle cells arising in the dermis as a multinodular ulcerative mass spreading into the subcutaneous and muscular plane ${ }^{1}$. It is a distinct cutaneous disease; metastasis is rare but it is highly aggressive and typically occurs in the trunk and extremities. Although, it can occur in all ages, highest number of cases is seen in second and fifth decades. This relates to its slow growing nature, where it occurs as a modest swelling slowly growing to a large mass which is the common presentation, hence ignored and left untreated for years.1,2 DFSP has an exceptional prognosis after complete resection but has a remarkable tendency to recur. It is a distinct superficial lesion not requiring extensive imaging work-up for its treatment. When an MR imaging is advised, it is usually in an atypical primary lesion which might be immensely large or in recurrent diseases. Recurrence might be common but metastasis namely bony extension is a rarity in such cases. MR imaging is a useful tool in identifying the location and extent of Dermatofibrosarcoma protuberans. ${ }^{1}$

Dermatofibrosarcoma protuberans is a rare soft tissue tumour showing moderate to low grade malignancy and is known to recur. MRI shows peculiar features specific to this type of soft tissue lesion. We present an interesting case of recurrent Dermatofibrosarcoma protuberans of foot in a 65-year-old woman who underwent excision of a similar lesion in the past. Our case demonstrated deep invasion directly involving the bone. MRI scan was performed, definite location and extent of the lesion was given, the patient was operated for the same and the excised sample was sent for histopathological examination which confirmed the tumour to be Dermatofibrosarcoma protuberans.

\section{PRESENTATION OF CASE}

A 65-year-old female presented with a swelling in her left lower limb on the medial side of calcaneal region of foot. There was history of a similar indolent swelling in the foot and wide local excision was performed one year back. The current lesion was initially small but gradually progressed to a large ulcerating mass associated with pain, involving the entire foot. It was an irregular and firm, hard in consistency and overlying skin was stretched with no discharge from the previous scar-line which appeared healthy and there was no wound gap. MR imaging was performed as there was history of previous soft tissue malignancy and this could be recurrence of same. On MRI, there was a large altered signal intensity soft tissue mass noted in the medial aspect of foot involving medial malleolar and sole region appearing iso to hyperintense on T2WI, hypo intense on T1WI and heterogeneously hyper intense on PD (proton density). There was interspersed multiple universally hypo intense bands within the mass lesion, it was seen to be present in the plantar fascia and underlying musculature namely quadratus plantae muscle, abductor hallucis muscle, plantar Apo neurosis extending superiorly up to Kager's fat pad. The mass was extending into the calcaneum bone causing its disruption showing adjacent scalloped margins with minimal reactive changes.
Corresponding Author:

Dr. Pratap Singh Hanuman Parihar.

Department of Radiodiagnosis, DMIMS,

Sawangi (Meghe), Wardha,

Maharashtra, India.

E-mail: drphparihar@gmail.com

DOI: $10.14260 / j e m d s / 2020 / 603$

How to Cite This Article:

Patwa P, Parihar PSH, Singh RK, et al. Atypical bony extension in recurrent dermatofibrosarcoma protuberance of foot. J Evolution Med Dent Sci 2020;9(37):27732775, DOI: 10.14260/jemds/2020/603

Submission 10-06-2020,

Peer Review 05-08-2020,

Acceptance 11-08-2020,

Published 14-09-2020.

Copyright (C) 2020 JEMDS. This is an open access article distributed under Creative Commons Attribution License [Attribution 4.0 International (CC BY 4.0)] 
A provisional diagnosis of recurrent DFSP with local extension into the bone was made. The lesion was resected with below knee amputation and the sample was sent for histopathological examination which showed an ulcerative growth on the proximal margin with an unremarkable epithelial lining and the deeper tissues were composed of fibro collagenous adipose stroma. The distal margin of the specimen was outlined by hypertrophied squamous epithelium with keratosis and the deeper tissue was similar as proximal containing fibro collagenous in nature with prominent vasculature.
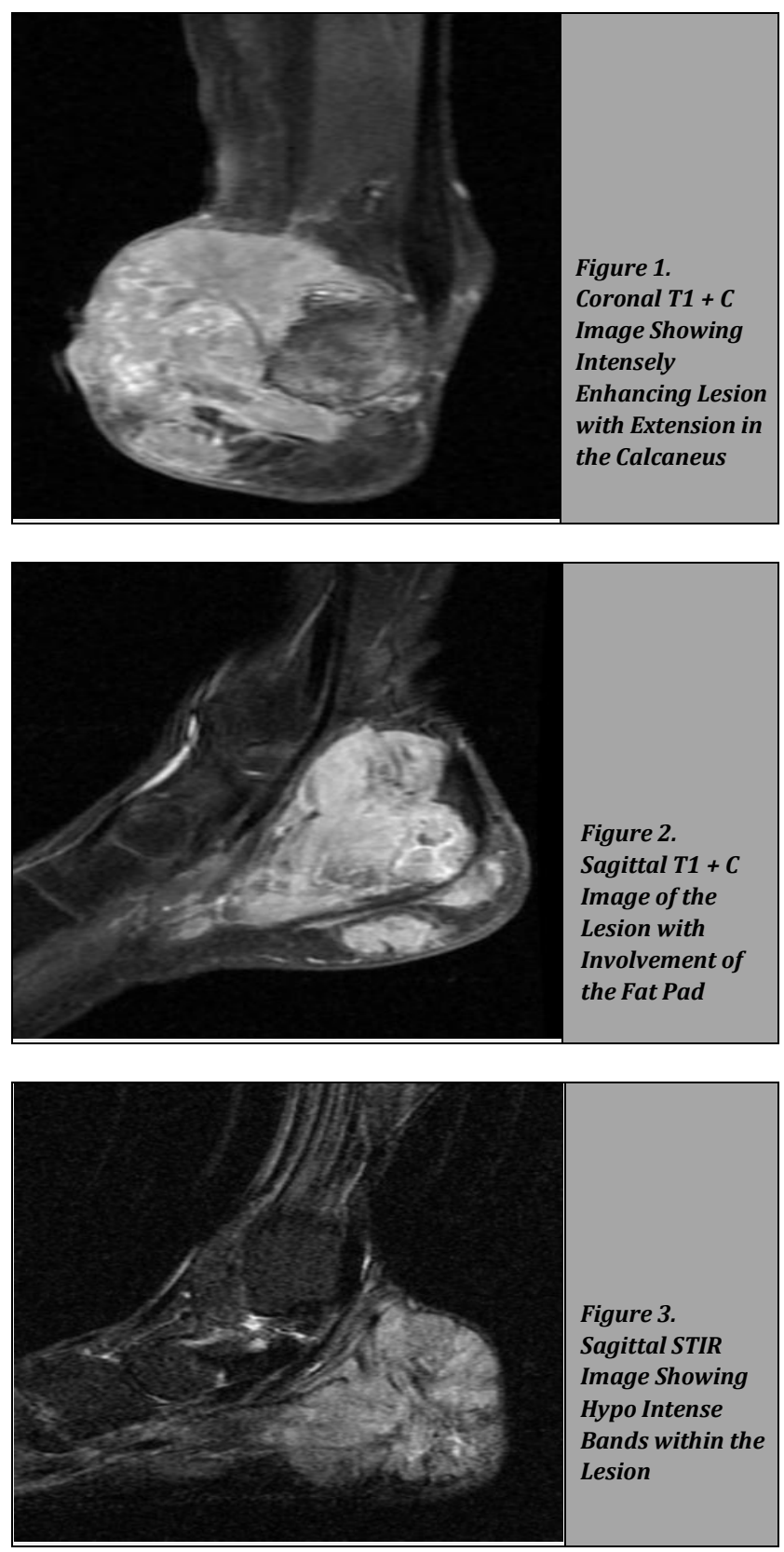

DISCUSSION

The main causative factor of DFSP is the presentation of prior traumas, including surgical scars, trauma scars, burns, radiodermatitis and insect bites with the most common anatomical site being the trunk, next common location is the proximal extremities (particularly the legs) and the head and neck areas $^{3}$. Majority of the cases of DFSP are usually small and superficial slow-growing asymptomatic plaque like lesion explaining late stage presentation to the doctor and diagnosis may be suspected based on its clinical appearance, while physical examination may assess the extension of the tumour. ${ }^{4}$ It initially presents as a heliotropic reddish-brown or pink plain plaque with a hard consistency; hence misinterpreted as a hypertrophic scar. With time, the neoplasm diffusely infiltrates in the deep layers of the skin and dermis, causing development of multiple nodules, that are adherent to the surrounding tissues, including the subcutaneous fat, fascia, muscle, sometimes periosteum and bone. ${ }^{3}$ These tumors rarely present as an area of atrophy or small subcutaneous nodules rather than a plaque. A prior history of trauma is reported in few cases and larger lesions can ulcerate, bleed, and become painful. The tumor is characterized histologically by surface bound CD34 and the absence of factor XIII a, which are used to differentiate it from other soft-tissue tumors mainly dermatofibroma. Coming on to imaging modalities for diagnosis of the lesion, both MRI and computed tomography (CT) is used to define the underlying structure of lesion. ${ }^{5}$ Magnetic resonance imaging (MRI) is fruitful for the estimation of the tumor invasion, mainly in cases of large tumors or large recurrent lesions. MRI provides valid information about the accurate position of the tumor, while it is an effective method for the differential diagnosis in cases of tumors that occur in an atypical site ${ }^{4}$. MRI has thus stood out as the modality of choice for margin definition in a planned operative procedure for DFSP because of its increased resolution as compared with CT. MRI also outshines CT in its utility for imaging DFSP because of the ability to procure images with unmatched imaging protocols: T1WI, T2WI, proton density fat saturation (PD fat-sat), and short tau inversion recovery (STIR). DFSPs are usually hypo intense to fat on T1-weighted images, hyper intense to is intense to fat on T2-weighted images and hyper intense on both PD fat-sat and STIR. Specialized techniques such as PD fat-sat and STIR are frequently used to accentuate the pathology on MRI. Using these sequences, MRI has been found to be useful in identifying atypical cases, the extent of tumor spread, and recognizing depth and involvement of crucial structures. The use of MRI is now proposed in DFSP for the following situations: large tumor size, tumors with a suspected deeper component, recurrent tumors, critical anatomic locations, and re-excision of DFSPs with positive surgical margins. ${ }^{5}$ DFSP is a locally invasive fibro-histiocytic tumor that recurrence even after wide local excision with a safety margin of 3 to $5 \mathrm{~cm}$. This is accredited to the fact that DFSP extends beyond the assessed clinical margins, spreading locally in the dermis to subcutaneous tissues and muscles.

Our case also demonstrates a deeper involvement directly extending to the calcaneum. As such deep invasion of the tumor with infiltration into the underlying bone is not frequent. This could to some extent be due to the fact that imaging is an uncommon part of the pre-operative workup in such patients. ${ }^{6}$ Medical as well as surgical treatment options exist for DFSP. Chemotherapeutic agents are used against the tyrosine kinase receptors which are often found to be overexpressed in this disease condition and for locally invasive DFSP, a surgical technique of wide excision with margins of 2$3 \mathrm{~cm}$ is the gold standard practice. Radiotherapy has also been used in the management of DFSP principally as an adjuvant to 
surgery and chemotherapy in cases where there is a high likelihood that the lesion was not completely removed through surgery. 5

\section{CONCLUSIONS}

As we have discussed, DFSP is a very rare but prospective malignant soft-tissue tumor with extensions to nearby structures and a timely detection of the disease can significantly decrease patient morbidity and mortality and is thus critical. Knowledge of the wide range of peculiar MR imaging features of dermatofibrosarcoma protuberans is, therefore, valuable. It is a key to accurate preoperative assessment and aids in the diagnosis of atypical or difficult cases.

Financial or Other Competing Interests: None.

\section{REFERENCES}

[1] Torreggiani WC, Al-Ismail K, Munk PL, et al. Dermatofibrosarcoma protuberans: MR imaging features. AJR Am J Roentgenol 2002;178(4):989-93.

[2] Asiri M, Moghazy KM, Alsaif HS, et al. Dermatofibrosarcoma protuberance: a case report and review of literature. Biomedical Research 2008;19(2):141-4.

[3] Stivala A, Lombardo GA, Pompili G, et al. Dermatofibrosarcoma protuberans: our experience of 59 cases. Oncol Lett 2012;4(5):1047-55.

[4] Stamatakos M, Fyllos A, Siafogianni A, et al. Dermatofibrosarcoma protuberans: a rare entity and review of the literature. J BUON 2014;19(1):34-41.

[5] Wales C, Caravaglio JV, Radi M, et al. A rare case of dermatofibrosarcoma protuberans of the forefoot. Radiol Case Rep 2016;11(2):102-5.

[6] Garg MK, Yadav MK, Gupta S, et al. Dermatofibrosarcoma protuberans with contiguous infiltration of the underlying bone. Cancer Imaging 2009;9(1):63-6. 